How to reference this article

Corea, A. (2019). Donne "compositrici" di balli pantomimi in Italia fra Sette e Ottocento. Italica Wratislaviensia, 10(2), 175-192.

DOI: http://dx.doi.org/10.15804/IW.2019.10.1.24

\author{
Annamaria Corea \\ Università degli Studi di Roma "La Sapienza" \\ annamaria.corea@uniroma1.it \\ ORCID: https://orcid.org/0000-0001-5670-9121
}

\title{
DONNE "COMPOSITRICI" DI BALLI PANTOMIMI IN ITALIA FRA SETTE E OTTOCENTO
}

\section{WOMEN "COMPOSERS" OF PANTOMIME BALLETS IN ITALY BETWEEN THE $18^{\mathrm{TH}}$ AND $19^{\mathrm{TH}}$ CENTURIES}

\begin{abstract}
This paper describes some aspects of the lives and artistic activities of Anna Binetti, Maria De Caro, and Giovanna Campilli, "prima ballerinas" who lived between the second half of the $18^{\text {th }}$ century and the beginning of the $19^{\text {th }}$ century. This research aims to highlight how these personalities practised as choreographers at a time when this profession was principally a male prerogative. Through the analysis of ballet programmes and contemporary sources, this paper investigates an area that has been little explored in the studies of the history of dance that is, the female presence as an "artistic subject" in the theatre of Italian dance. Thus, this phenomenon is contextualised in the broader cultural landscape of the time and focuses on three case studies of women choreographers who, whilst isolated in their extraordinariness, often converged significantly in their performance venues, in particular in Venice and Naples, and in personalities such as Jean-Georges Noverre and Maria Carolina of Austria. What is most interesting is that this approach highlights possible relationship networks and, thus, may open new avenues of research.
\end{abstract}

Keywords: Anna Binetti, Maria De Caro, Giovanna Campilli, $18^{\text {th }}$ - and $19^{\text {th }}$-century Italian ballet, ballet programmes/librettos 
$\mathbf{R}$ ipercorrendo a grandi linee la storia della danza occidentale, emerla donna in quanto "soggetto artistico" si afferma pienamente nel XX secolo e soprattutto nell'ambito di nuove forme come la danza libera, poi moderna e contemporanea, ricoprendo oltre al ruolo di interprete anche quello di coreografa. Nel genere di tradizione del balletto, pur occupando un posto fondamentale come ballerina, spesso responsabile della creazione dei propri soli, le donne non hanno ricoperto competenze specificamente autoriali "accreditate" fino al primo Novecento, eccetto qualche rara eccezione. Concentrandoci su alcuni casi straordinari, situati tra la fine del Settecento e i primi decenni dell'Ottocento, si tenterà di mettere in luce la presenza di artiste che hanno rappresentato con la loro attività di coreografe, anche se più o meno sporadica, un'eccezione rispetto alle pratiche dell'epoca; pratiche d'arte che vedevano l'uomo al centro delle responsabilità produttive dello spettacolo coreutico, sia nel campo organizzativo che creativo ${ }^{1}$.

Possiamo far risalire i primi significativi esempi di "autorialità" femminile, ascrivibile al genere ballettistico, a due note danzatrici francesi - Françoise Prévost (1680-1741) e Marie Sallé (1707-1756) - che si ricordano come pioniere di nuove modalità sceniche e drammaturgiche all'insegna di una danza espressiva. Prévost, danzatrice all'Opéra, è famosa per la sua recitazione muta ed eloquente al tempo stesso, in una scena dell' Orazio di Corneille, quella dell'uccisione di Camilla (atto IV, scena 5), rappresentato al castello di Sceaux nel 1714 grazie all'iniziativa della duchessa del Maine. L'anno seguente, ricreò attraverso l'interpretazione di dodici danze dell'epoca una sorta di narrazione per quadri focalizzata sull'amore nelle sue varianti di caratteri e situazioni, all'in-

1 Un altro fondamentale ambito lavorativo, come la formazione del danzatore, era affidato ufficialmente agli uomini, i maîtres de ballet, i quali sin dalla tradizione cortese avevano anche mansioni di coreografi. Resterà questa la situazione in Italia, con sporadiche eccezioni, come l'affidamento a Paolina Elisabetta Cholat Naley Neuville della sezione di perfezione femminile alla scuola del Teatro di San Carlo di Napoli (Maresca, 1997, pp. 97-98). Alla scuola del Teatro alla Scala di Milano, le donne affiancavano spesso i mariti nell'insegnamento; notiamo inoltre che solo nel 1902 a Caterina Beretta fu assegnata la carica di direttrice (Scafidi, 1998, pp. 24-25). 
terno del divertissement composto per lei da Jean-Ferry Rebel, Les Caractères de la danse (1715), riuscendo «ad investire la danza accademica di un colore e di un'intensità espressiva totalmente nuovi» (Pappacena, 2009, p. 76). Allieva di Prévost, Sallé sbalordì per le sue esibizioni fuori dagli schemi. Nel 1729 all'Opéra di Parigi, a conclusione dell'Alceste di Lully-Quinault, era apparsa in un pas de deux con un abito comune e senza la maschera; qualche anno più tardi, nel 1734, al Covent Garden di Londra, aveva presentato due balletti di sua invenzione, Pygmalion e Bacchus et Ariane, la cui portata innovativa fu subito registrata dal recensore del "Mercure de France" che a proposito di quest'ultimo puntava l'attenzione sulle strategie coreutiche finalizzate all'espressività del corpo: «si tratta delle espressioni e dei sentimenti del dolore più profondo, della disperazione, del furore, dell'abbattimento; in una parola, tutti i grandi movimenti e la declamazione più perfetta, attraverso passi, attitudes e gesti, per rappresentare una donna abbandonata da colui che ama» (Ibidem, p. 82). Si informava inoltre che nel balletto Pygmalion, ispirato alle Metamorfosi di Ovidio, «ella ha osato apparire in questa scena senza panier, senza gonna, senza bustino, e con i capelli sciolti, e senza alcun ornamento in testa; indossava, con il corsetto e la sottana, solo un semplice vestito di mussola drappeggiato e sistemato a modello di una statua greca» (Ibidem). La scelta delle espressioni e dei costumi ${ }^{2}$ in base a una precisa coerenza drammatica fa della Sallé una precorritrice riconosciuta del balletto narrativo che si svilupperà a partire dagli anni quaranta del Settecento con le prime forme di ballets-pantomime e avrà la sua consacrazione teorica con il trattato di Jean-Georges Noverre, Lettres sur la danse et sur les ballets (prima edizione: 1760).

Spostando lo sguardo all'Italia, la presenza documentata di donne coreografe nel Settecento è molto rara. I dati raccolti attraverso i cataloghi bibliotecari e specifici cataloghi di libretti per musica, incrociati

2 Anche nell'ambito del costume teatrale le donne riuscirono a ritagliarsi un margine di creatività, influenzando in taluni casi la moda del tempo e assorbendo esse stesse gli stimoli dagli altri ambiti artistici. Un esempio fra tanti è Marie-Madeleine Guimard (1743-1816) che aveva modificato l'abito di scena, eliminando le sottogonne e creando un modello caratterizzato da due gonne sovrapposte diffusosi con successo nella moda parigina. Cfr. Nordera, 2007 e Bennahum, 2004. 
con le cronologie dei teatri interessati, ci dicono che nella prima metà del secolo le donne creano i balli per lo più in coppia con un uomo ${ }^{3}$; nei tre decenni di comparsa e sviluppo del ballo pantomimo, ovvero dagli anni cinquanta a tutti gli anni settanta, non risultano presenze femminili; dal 1780 al primo ventennio dell'Ottocento sono stati individuati invece tre nomi di donne "compositrici", Anna Binetti, Maria De Caro e Giovanna Campilli. Nei primi due casi si tratta di danzatrici conosciute, anche se oggi poco si sa della loro vita e attività, mentre la Campilli è una di quelle figure, come tante e non solo femminili, di cui si è persa completamente traccia ${ }^{4}$. I tre casi si collocano in un periodo di consolidamento del ballo pantomimo in quanto genere di spettacolo entr'acte all'interno delle opere in musica o in prosa. In questi balli, ispirati a uno spettro molto ampio di soggetti - mitologico, storico, tragico, comico ecc. - in cui sezioni interamente pantomimiche si affiancavano a sezioni di pura danza eseguite secondo la tecnica accademica francese, la scrittura drammaturgica e coreografica spettava ai cosiddetti "compositori di balli", i cui nomi comparivano sul frontespizio del programma quali principali autori dello spettacolo 5 .

3 A questa altezza cronologica i balli non hanno ancora una struttura narrativa e per tale motivo sono privi di titolo. Questi i dati estrapolati dal catalogo di Claudio Sartori, sulla prima parte del XVIII secolo: 1709, Ferrara, fiera di maggio, balli di M.me de Menemare e monsieur de Raymond; 1713, Palazzo Reale di Napoli, ottobre, balli di Gio. Battista Du Fort e Anna Daufin; 1716, Teatro Obizzi di Padova, carnevale, balli di messieurs Jean et André Gallo, de Madame Mariane et monsieur Paule de Corsèl; 1718, Teatro Tron di San Cassiano di Venezia, carnevale, balli di Susanna Dentis (per le opere Antigona e Farnace); 1732, Teatro Nuovo di Napoli, direttore dei balli Rocco Luongo insieme a Francesca Melano e compagne; 1738, Teatro di Piazza in Vicenza, balli di Maria Viganò e Andrea Alberti; 1750, Teatro Omodeo di Pavia, carnevale, balli di Lodovico e Anna Ronzi.

4 Sollecitazioni a indagare questo ambito sono venute da Pappacena, 2017, p. 15. Ringrazio Flavia Pappacena per tutti i suggerimenti e gli scambi durante la redazione di questo saggio.

5 I programmi del ballo, stampati all'interno del libretto d'opera o come opuscoli a parte, sono fonti di primaria importanza per la danza di questo periodo, attraverso cui è possibile reperire diverse informazioni sulla messinscena: luogo, anno, stagione; dati relativi alla compagnia (nomi e cognomi dei componenti, il loro rango, ovvero la competenza tecnica, il ruolo ricoperto); nomi del costumista e dello scenografo, 
Nella stagione di Carnevale del 1780, al Teatro Giustiniani di San Moisè a Venezia, andava in scena La filosofia delle donne, «balletto allegorico, inventato e posto in su la scena da Madama Binetti» (La filosofia delle donne, 1780, p. 1), danzatrice conosciuta per essere stata legata, sia nella vita privata che in quella artistica, a Charles Le Picq (1744-1806), uno dei più noti e prediletti allievi di Noverre, nonché riproduttore di molti suoi balli ${ }^{6}$. Binetti e Le Picq, che si erano conosciuti nella splendida corte del duca di Württemberg a Stoccarda (Winter, 1974, p. 170), dopo un tour di circa un lustro che li vede impegnati a Vienna, Varsavia e San Pietroburgo (Dorvane, 1997), fanno coppia fissa in diversi teatri italiani: al Teatro Nuovo di Padova (1769-1770), al San Benedetto di Venezia (1770, 1772, 1777-1778), al Teatro Ducale di Milano (1771-1773) e al San Carlo di Napoli (1773-1776), dove ebbero il merito - lui come "compositore di balli" ed entrambi come "primi ballerini seri" - di introdurre il genere del ballet d'action di stile noverriano (Croce, 1891 , p. 549) .

Notizie biografiche più dettagliate su Anna Binetti ricaviamo dalle memorie di Giacomo Casanova (1725-1798), che di lei scrisse con molta ammirazione. Veneziana, figlia del barcaiolo Ramon, sposò - per intercessione della sua protettrice Cecilia Valmarana - il ballerino e impresario francese Georges Binet, il quale aveva cambiato il suo cognome in Binetti, cosicché Anna «non fu costretta a francesizzare la sua natura

e meno spesso dell'autore delle musiche; la trama del ballo organizzata in atti e/o scene e descritta in modo dettagliato; le fonti letterarie utilizzate e in taluni casi opinioni del coreografo sul ballo rappresentato e sul ballo in generale.

6 Secondo Winter (1974), Binetti, rimasta vedova negli anni settanta, avrebbe sposato Le Picq, molto più giovane di lei, conosciuto a Stoccarda. Nella voce di Christout (1959) ci sono diverse inesattezze che riguardano proprio la Binetti: si suppone che sin dagli anni sessanta sia la moglie di Le Picq e che abbia francesizzato il suo nome in Binet, come era usuale.

7 Altro aspetto relativo alla condizione delle professioniste, che qui non possiamo approfondire, è quello economico. Il contratto di Le Picq a Napoli come compositore di balli prevedeva l'ingaggio di una partner «a sua elezione, ed a sue proprie spese». Questa modalità di scrittura, in linea con quanto succedeva altrove nella penisola, prevedeva un solo compenso al ballerino maschio che il più delle volte era affiancato dalla moglie o dalla sorella (Onesti, 2016, p. 108). 
veneziana che le permise di mostrare il suo temperamento in parecchie avventure che la resero celebre» (Casanova, 1965, vol. I, pp. 690-691) ${ }^{8}$. Conosciuta a Venezia, la donna «incatenò il cuore» (Ibidem, p. 690) di Casanova già dal 1747 e divenne negli anni una cara amica, la sua più vecchia conoscenza felicemente ritrovata nei viaggi a Stoccarda (1760), Londra (1763), Varsavia (1765), alcune fra le capitali europee della danza. Dai Mémoires di Casanova emerge non solo un ritratto di donna «sempre giovane» (Ibidem, p. 691), dalle «incantevoli attrattive» (Idem, 1965, vol. III, p. 449), di cui si sarebbe potuto innamorare, «attrattive possenti in tutta la persona» (Idem, 1965, vol. V, p. 269) come il «brio che rapiva la compagnia» (Ibidem), ma anche di donna indipendente, che viveva autonomamente e «a modo suo» (Idem, 1965, vol. I, p. 691), frequentando diversi amanti e cercando di stare il più possibile lontana da un marito irresponsabile che aveva il vizio del gioco ed era solito pagare i debiti con il denaro e i beni della moglie. Dopo una carriera lunga nelle piazze più prestigiose d'Europa, la Binetti tornò stabilmente nella sua città, dove nel 1778 danzò ancora il ruolo principale nel ballo $R i$ naldo di Onorato Viganò al San Benedetto di Venezia, ritirandosi dalle scene subito dopo, e restando attiva come maestra di danza e impresario teatrale (Idem, 1965, vol. VII, p. 449) ${ }^{9}$.

La circoscritta esperienza di compositrice di balli, di cui conosciamo solo due titoli, si situa quindi alla fine della carriera. Dall'Avviso al pubblico di Venezia, che apre il programma del ballo La filosofia delle donne ${ }^{10}$, veniamo a conoscenza di un suo primo ballo dal titolo Gelosie

${ }^{8}$ Binetti, fra le altre avventure, fu la causa del duello di Casanova con il conte Francesco Branicki, avvenuto a Varsavia nel 1766, su cui pubblicò Il duello ovvero Saggio della vita di G. C. veneziano, in Opuscoli miscellanei (Venezia, giugno 1780).

9 Dalle notizie fornite da Casanova possiamo desumere che Binetti fosse nata intorno ai primi anni venti del Settecento e scomparsa fra il 1784 e il 1786. A partire dal 1786 Le Picq si lega alla ballerina Gertrude Rossi con cui parte per San Pietroburgo.

${ }^{10} \mathrm{Il}$ ballo è rappresentato fra il primo e il secondo atto del dramma giocoso Le teste deboli di Giovanni Bertati. Ad Anna Binetti fu assegnata la composizione del primo ballo, ad Alberto Cavas del secondo. Un ballo dallo stesso titolo viene riproposto nel 1784 al Teatro alla Scala e nel 1788 al Teatro di Reggio da Filippo Beretti. Questo era sprovvisto di un programma, come avveniva spesso con i balli comici, e quindi non possiamo affermare che esso fosse una riproduzione di quello precedente della Binetti. 
nel Serraglio. L'autrice inoltre sottolinea che «una tale impresa accettata da una Donna», ovvero la composizione di un ballo, ha «un certo aspetto di novità, che può soggiacere a de' pericoli» (La filosofia delle donne, 1780, p. 3). Se l'argomento allegorico di questo secondo ballo è di invenzione della stessa coreografa, il primo titolo rimanda al ballo da lei interpretato, La Gelosia del Serraglio, che Le Picq mise in scena al Regio-Ducale di Milano nel dicembre del 1772 come primo ballo nell'opera di Mozart Lucio Silla, riprendendolo a sua volta da quello di Noverre, Die fünf Sultaninnen, oder Die traurigen Wirkungen der Eifersucht, dato al Burgtheater di Vienna nel 1771 (Pappacena, 2015, p. 117) ${ }^{11}$.

Possiamo trovare un tratto comune fra questi due balli di Anna Binetti nella centralità conferita alla figura femminile, pur nella diversità del genere di riferimento: Gelosie nel Serraglio apparterrebbe al filone "esotico", improntato sulla spettacolarità dei colori e dei movimenti, mentre La filosofia delle donne a quello "allegorico", caratterizzato da un' ambientazione galante pastorale e agito su un registro mimico-comico. Completamente all'opposto le due comunità di donne rappresentate. Da una parte, nel mondo turco del ballo Gelosie nel Serraglio le due prescelte dell'harem si contendono il favore del Gran Sultano a suon di pugnale, con la partecipazione appassionata delle compagne che parteggiano per l'una o per l'altra, tentando invano di dare fine alla rissa. $L a$ filosofia delle donne è invece ambientato in un villaggio immaginario. La scena rappresenta un bosco e sul fondo un colle praticabile da cui villanelle «tutte vestite di nero, colle chiome incolte, e disordinate, e un cappello in capo. Discendono (...) con una serietà comica caricata» ( $L a$ filosofia delle donne, 1780, p. 8). L'azione sembrerebbe procedere per lazzi - come si specifica nel programma: «leggendo comicamente, l'una

Certo è che fosse una prassi consolidata quella di riprodurre i balli, attingendo al vasto contenitore di soggetti estesi in forma di programma.

${ }^{11}$ Il primo ballo di Noverre su questo soggetto, peraltro molto in voga nel Settecento già con Hilverding, fu dato a Lione nel 1758 e si intitolava Les Jalousies ou Les Fêtes du Sérail; nel suo continuo rimando alla pittura, il coreografo ne scrive già nella VI lettera dell'edizione del 1760 del suo trattato, a proposito dell'uso dei colori con le loro gradazioni e combinazioni. 
di stupore, l'altra d'orrore, l'altra di giubilo» (Ibidem, p. 9) - attraverso cui le fanciulle esprimono la loro dedizione allo studio e l'indifferenza verso i gesti di affetto dei giovani. I protagonisti sono Cefisa, ricca pastorella «dedita alle Scienze, e sprezzante d'amore» (Ibidem, p. 5), e l'innamorato Tirsi, da lei non corrisposto. Lei insensibile, lui disperato tanto da essere sul punto di trucidarsi davanti al tempio di Apollo, che lo rassicura e intercede a suo favore. Mentre le fanciulle, opportunamente ferite dalla freccia di Cupido, subito «s'abbandonano a' loro amanti» (Ibidem, p. 10), Cefisa è pronta a lottare con tutta se stessa per difendere il suo status di donna insensibile al genere maschile, si scaglia contro le coppie danzanti per dividerle e intraprende una vera e propria lotta con Cupido che la rimprovera di essere gelosa dell'amore altrui. Non solo Cefisa respinge con ira Tirsi, quando Cupido minaccia di ferirla, «gli strappa l'arco, lo spezza, e lo scaglia a terra» (Ibidem, p. 11), ferisce addirittura il dio con una sua stessa freccia, consentendogli di fingere di essere sul punto di morire. All'apparente trionfo di Cefisa segue il coup de théâtre che scioglie la trama: Apollo, vestito con un abito nero da filosofo, attira Cefisa mostrandole un libro, ha così modo di ferirla e ottenere la sottomissione della donna che quindi viene ridicolizzata davanti a tutti gli astanti. Nel finale si esplica quindi il senso allegorico del ballo: la lezione che esso ci vuole dare è il richiamo della donna a un ordine sociale che la vedeva relegata in una dimensione privata, dedita non tanto allo studio e all'atto del filosofeggiare, quanto all'amore coniugale e alla famiglia.

Di tutt'altro genere è il successivo ballo rintracciato, a firma di una donna: Oscar e Malvina «Ballo Eroico Pantomimo In cinque Atti, composto e diretto da Mad. Maria De' Caro, Prima Ballerina» (Oscar e Malvina, 1797, p. 21) e rappresentato al Teatro Venier di San Benedetto nel 1797, a pochi mesi dalla caduta della Serenissima ${ }^{12}$. L'elemento in comune fra quest'ultimo ballo e quello di Anna Binetti è Ve-

12 Il soggetto del ballo ebbe una buona fortuna; fu rappresentato al Teatro della Pergola di Firenze nel 1798 da Giovan Battista Checchi; al Teatro Nazionale di Torino nel 1801 da Antonio Landini (in cui "prima ballerina seria" era la stessa De Caro); tutte queste versioni ballettistiche hanno preceduto il dramma per musica omonimo di Fidanza/Sampieri al Teatro Re di Milano del 1816. 
nezia, città particolarmente vivace e produttiva, dove si era sviluppato un ampio dibattito teorico sul teatro musicale e sul ruolo del ballo pantomimo come entr'acte, portato avanti tra gli altri da Francesco Milizia (1771), Ange Goudar (1773) e Stefano Arteaga (1785). Nel 1794 su uno dei numerosi periodici editi nella laguna, la "Gazzetta Urbana Veneta", comparve a puntate la prima traduzione italiana delle Lettres di Noverre, sebbene in forma anonima e non integrale, e le "libere" traduzioni di alcune voci dell'Encyclopédie relative alla danza (Ruffin, 1998, pp. 35-58) ${ }^{13}$. Città dinamica e moderatamente aperta, Venezia accolse la letteratura d'oltralpe e la cultura illuminista, anche attraverso due figure femminili come Elisabetta Caminer Turrà (1751-1796) e Giustina Renier Michiel (1755-1832) che in questa direzione contribuirono significativamente con i loro salotti e l'intensa attività culturale. La prima avrà un ruolo fondamentale nel giornale fondato dal padre "L'Europa letteraria" e in quello da lei stessa fondato e diretto, il "Giornale enciclopedico" (poi "Nuovo Giornale enciclopedico"), oltre che nella diffusione in quanto traduttrice di pièce francesi contemporanee; la seconda fu autrice fra l'altro della prima traduzione italiana di tre drammi shakespeariani - Otello, Macbeth e Coriolano - basata sulle versioni originali inglesi ${ }^{14}$. Entrambe furono legate a Melchiorre Cesarotti (1730-1808), anch'egli figura chiave della cultura italiana del secondo Settecento, che qui ricordiamo per la traduzione in endecasillabi dei Fragments of Ancient Poetry di James Macpherson, le Poesie di Ossian, da cui il soggetto del ballo Oscar e Malvina trae ispirazione. Sembrerebbe questo il primo ballo rappresentato in Italia, basato su tematiche ossianiche, sull'onda delle varie

13 Delle quindici lettere solo otto vengono tradotte sul bisettimanale. È rimasta inedita la traduzione di Domenico Rossi del 1778 per un'edizione napoletana, il cui manoscritto si trova nel fondo Fornaroli della New York Public Library.

14 Si tratta dei tre volumi usciti a Venezia (eredi Costantini) tra il 1798 e il 1800 con il titolo Opere drammatiche di Shakespeare volgarizzate da una dama veneta. È importante ricordare che in questi anni novanta del Settecento due scritti fondamentali per la storia delle donne furono pubblicati: Dichiarazione dei diritti delle donne e della cittadina di Olympe de Gouge (Parigi, 1791) e Rivendicazione dei diritti delle donne di Mary Wollstonecraft (Londra, 1792). 
edizioni cesarottiane che si susseguirono a partire dal 1763, e di una librettistica via via sempre più interessata a nuovi soggetti legati al mondo celtico, al gusto per il primitivo e il tenebroso e alla riscoperta del Medioevo e di Shakespeare, che sono indicativi di una emergente sensibilità romantica (Piperno, 2016, pp. 137-159) ${ }^{15}$.

Maria De Caro, che aveva esordito come "prima ballerina seria" proprio nel Teatro di San Moisè nel 1785, era anche lei legata direttamente al maestro Noverre, come Binetti e Le Picq; è infatti una delle sue più rinomate pupille, oltre che allieva di Armand Vestris; attiva per un breve periodo a Venezia, trascorse circa sette anni a Londra dove raggiunse un notevole successo anche economico, poi a Parigi, sempre insieme alle sorelle, tornando a esibirsi in diverse piazze italiane, come Venezia, Firenze, Milano, Napoli, poi a Vienna, dal 1796 fino al ritiro dalle scene avvenuto nel 1806 (Costa, 1987) ${ }^{16}$. Il ballo che la vede coreografa è rappresentato poco dopo il suo rientro in Italia. Il programma non contiene però un Avviso al pubblico e il singolo episodio del ciclo epico del leggendario bardo irlandese, Ossian, relativo a suo figlio Oscar, è riassunto piuttosto schematicamente in cinque brevi atti, da cui ricaviamo informazioni soprattutto riguardo all'ambientazione. Particolarmente ossianica doveva essere la scena dell'atto III in un bosco, in cui avviene il rapimento della protagonista Malvina - sposa del guerriero Oscar e figlia del re Toscar, interpretata dalla stessa De Caro - da parte dell'usurpatore Cairbar; suggestiva anche la scena dell'atto IV nella grotta in cui vengono imprigionati la fanciulla e il sopraggiunto Oscar. L'azione si scioglie nell'atto V con una scena di combattimento fra Oscar e Cairbar e con il trionfo della

15 Segnalo un libretto di ballo stampato a Londra nel 1791, The airs, duets, choruses, and argument of the new ballet pantomime (taken from Ossian) called Oscar and Malvina; or the hall of Fingal as performed at the theatre-Royal Covent-Garden, da cui Maria De Caro avrebbe potuto trarre ispirazione, trovandosi all'epoca in quella stessa città (McCleave, 2011, p. 101).

${ }_{16}$ Maria era la primogenita, ma non si conoscono le date di nascita e morte, le altre si chiamavano Maddalena e Francesca, mentre dell'ultima De Caro si conosce solo l'esistenza. Una variante del cognome è Del Caro, a cui si aggiunge il cognome del marito Narducci. 
virtù cavalleresca dell'eroe che rende possibile il ricongiungimento dei protagonisti Oscar e Malvina.

L'attività di Maria De Caro come coreografa è circoscritta a questa singola esperienza, peraltro poco conosciuta; molto nota è invece la sua attività di danzatrice, come detto, suggellata da un'incisione di Giacinto Maina del 1801, pubblicata da Giuseppe Sardi a Trieste (Winter, 1974, p. 216), che la ritrae su tre quarti di punta e in un atteggiamento del corpo "in moto" in cui gesto e danza sembrano fondersi e che testimonia l'evoluzione della tecnica francese verso un dinamismo e un virtuosismo fino ad allora inesplorati. Movimenti più ampi sia in larghezza che in altezza sono possibili anche grazie a una graduale riforma del costume affermatasi in pieno neoclassicismo con il successo del vestito alla greca. In questa immagine, l'abito stile impero che indossa la ballerina ci rimanda al legame fra Maria De Caro e l'inglese Emma Hamilton, residente a Napoli fra il 1786 e il 1799 insieme al marito, ambasciatore presso la corte borbonica. Proprio nella sua casa la Hamilton si esibiva in attitudes ispirate al mondo dell'antichità che eseguiva vestita con leggere tuniche di mussola, moda che contribuì a diffondere nell'ambiente artistico. Al Teatro San Carlo di Napoli, la De Caro fu ingaggiata grazie alla segnalazione di lady Hamilton, che l'aveva raccomandata alla regina Maria Carolina d'Asburgo-Lorena, come si evince dal carteggio fra le due donne (Palumbo, 1877). È in questa intrigante trama di relazioni che pure si inserisce - anche se in modo meno diretto - la terza coreografa di cui tratteremo, Giovanna Campilli.

A differenza delle colleghe Binetti e De Caro, Giovanna è oggi pressoché sconosciuta nonostante sia stata "prima ballerina" nei più prestigiosi teatri della penisola, tanto da ispirare già nel 1801 un affermato compositore di opere serie come Sebastiano Nasolini a dedicarle un assolo ballabile. Il primo dato relativo alla sua carriera di danzatrice si riferisce al Teatro Nuovo di Padova dove fu "prima ballerina" nel 1792, in coppia con Giuseppe Bartolomei, nei balli Bacco e Arianna e La fiera di Calè. Compare nello stesso cast, nel rango di «primo ballerino di mezzo carattere fuori de' concerti», Leopoldo Campilli, presumibilmente suo padre, attivissimo anche come core- 
ografo sin dal 1780 nei teatri del circuito veneziano, San Benedetto, San Samuele, San Cassiano e San Moisè (Massaro, 1985, pp. 251-252). La vicinanza di Campilli padre con coreografi e danzatori di scuola noverriana, come Antonio Terrades e Maria De Caro, che nel 1785 egli stesso aveva diretto al San Moisè, insieme alla competenza nel ricoprire ruoli di "primo grottesco" e "grottesco fuori dei concerti", tipici della scuola italiana (Sartori, 1990-1994; Wiel, 1897), fanno supporre che la formazione di Giovanna sia avvenuta su questo doppio registro italo-francese. I primi anni di carriera, gli anni novanta, sono segnati dalla collaborazione - prima al San Moisè (1793-1794), poi al Teatro Regio di Torino (1798) - con Urbano Garzia, coreografo produttivo e maestro di mimica nella scuola di ballo del Teatro alla Scala dal 1813 al 1823 (Scafidi, 1998, p. 16). Nel primo decennio dell'Ottocento, Giovanna come "prima ballerina" lavorò con Gaetano Gioia e Francesco Clerico, rispettivamente al San Carlo di Napoli (1801-1804) e alla Scala di Milano (1805-1806), a confronto con due autorevoli personalità, entrambi particolarmente prolifici, ma animati da una diversa concezione dello spettacolo coreutico e dello stile pantomimico. Se i balli di Gioia possono ascriversi a quel genere denominato da Carlo Ritorni «coreodramma», ovvero «canto muto», il cui esimio rappresentante - sempre secondo questo autore - fu il coevo Salvatore Viganò, i balli di Clerico erano delle «pantomimotragedie», drammi muti, caratterizzati soprattutto dalle forme dell'assolo e dei duetti, e dall'uso di una preponderante gestualità di tipo noverriano, quindi "recitata", in contrapposizione alla gestualità ritmata tipica del coreodramma, in cui simultaneamente ai danzatori principali anche il corpo di ballo si animava in accordo alla musica catturando l'attenzione del pubblico per il dinamismo e la varietà del gioco visivo (Ritorni, 1838, pp. 32-42).

La Campilli fece esperienza di entrambe le modalità coreografiche. Ci chiediamo quindi quale sia stato il suo metodo e il suo stile quando qualche anno più tardi si approcciò alla composizione di balli pantomimi presso il Real Teatro Carolino di Palermo, per almeno tre stagioni a partire dal 1809, occupando al contempo il posto di "prima ballerina seria assoluta", in coppia con Pietro Campilli, presumibilmente suo fratello, 
noto per essere autore della prima traduzione italiana dell'importante Trattato elementare, teorico-pratico sull'arte del ballo (1820) di Carlo Blasis, edita a Forlì nel $1830^{17}$. Sono gli anni in cui la corte borbonica si trasferì a Palermo in seguito all'arrivo dei francesi a Napoli; un'altra domanda viene a questo punto spontanea: è possibile che la regina Maria Carolina d'Asburgo-Lorena, a cui si deve la denominazione del Teatro a partire dal 1799, si facesse promotrice della Campilli come già aveva fatto alla fine degli anni novanta, attraverso Emma Hamilton, con Maria De Caro al San Carlo? Insieme alla coppia Giovanna e Pietro, vi è il padre Leopoldo, che alterna con lei la responsabilità della composizione dei balli, come avviene nella stagione $1811 / 12^{18}$. Fu tuttavia Giovanna $\mathrm{a}$ iniziare le stagioni palermitane con balli per lo più di genere eroicotragico: Rossene e Osmano e Ippolita regina delle amazzoni (1809/10); Le smanie di Armida, Il mastino della scala e Zemira e Azor (1810/11) ${ }^{19}$.

I titoli di questi balli riecheggiano balli con lo stesso soggetto già rappresentati da altri coreografi e confermano una pratica molto diffusa all'epoca, ovvero l'utilizzo dei programmi del ballo come materiale di un repertorio condiviso che circolava fra le varie piazze della penisola senza la preoccupazione del plagio, visto che non esisteva ancora il diritto d'autore. In particolare, Il mastino della scala è un ballo di Giuseppe Traffieri in cui aveva danzato Leopoldo Campilli, reppresentato al San Benedetto di Venezia nel 1790. Mentre Zemira e Azor, tratto da una comédie-ballet di Marmontel, aveva il suo precedente nel ballo

17 È stato possibile reperire $\mathrm{i}$ dati relativi ai balli rappresentati al Teatro Carolino attraverso la HathiTrust Digital Library. Alcuni di essi si possono individuare attraverso alcuni database on line come quello dell'Università di Bologna, "Corago". Non vi è però traccia di questi balli nel catalogo unico centrale delle biblioteche italiane. Abbiamo incrociato i dati trovati con la cronologia curata da Ottavio Tiby (1956), che dei balli riporta solo i titoli, omettendo i nomi dei coreografi.

18 Per la stagione 1811/12 Leopoldo Campilli compone i seguenti balli: Gengis-Kan in Corea, Il finto morto, Venere benefica, Gli inglesi in Africa e Il convitato di pietra.

19 Non datati sono i seguenti balli rintracciati nella HathiTrust Digital Library di cui non è disponibile copia digitale, tutti «composti e diretti» da Giovanna Campilli: Nina, o sia, La pazza per amore, «ballo pantomimo sentimentale», Eduige di Witepsk, ossia, Il sottoterraneo, «ballo eroico-tragico», Li due ciechi che vedono chiaro, o sia, Lo sposo burlato, «ballo comico». 
omonimo di Clerico dato prima al San Samuele di Venezia (1783), poi alla Fenice (1797) e infine alla Scala (1805), in cui aveva danzato pure Giovanna Campilli.

Quando rientrò a Napoli, probabilmente sua città natale, Giovanna non si cimentò in nuovi titoli, tanto meno riprese un'attività continuativa come coreografa ${ }^{20}$. Riprodusse a distanza di diversi anni due balli rappresentati al Teatro del Fondo - Ippolita (1816) e Venere benefica (1827), continuando la sua carriera come "ballerina per le parti" o "mima", affianco a nomi di spicco come Marie Quériau e Louis Henry, in ruoli maggiormente adeguati all'età più avanzata che ricoprì all'incirca per tutti gli anni venti dell'Ottocento. «Nome caro alla danza, e con piacere e con stima ricordato da chiunque si rammenta quanta lode ella ritrasse altra volta nelle classiche composizioni pantomimiche del Napoletano Gioja» (“Giornale delle Due Sicilie", 20 giugno 1816, p. 3), la Campilli seppe svolgere adeguatamente la sua professione di compositrice di balli se l'Ippolita venne considerato un ballo «degno certamente di lode per scelta di argomento, per semplicità di condotta, per verità di caratteri, per disegno e bellezza di danze» benché «mancante di ogni prestigio di macchinismo» (Ibidem) e difettoso nella scelta dei costumi, non aderenti alla verosimiglianza storica. Doveva essere il personaggio di Ippolita uno degli ultimi a ricoprire come "prima ballerina seria", dotata com'era di una «rara abilità ${ }^{21}$ tecnica, prima di dedicarsi a ruoli essenzialmente mimici. Per quanto riguarda i danzatori, la recensione si focalizza però sugli astri nascenti, la coppia Adelaide e Salvatore Taglioni, mentre di Giovanna si scrive di «aver meritato nella composizione i suffragj da' conoscitori del vero ballo, e di non averne riportato nell'esecuzione minori da tutti quelli presso i quali è in pregio l'eccellenza della pantomima» (Ibidem). Difficile stabilire i modi e lo stile di una professione

20 Nell' Avviso contenuto nel programma di ballo della rappresentazione napoletana Venere benefica la coreografa si rivolge al pubblico di Napoli usando il termine «concittadini» (p. 9).

21 Lettera del 26 maggio 1802 conservata nel Fondo "Segreteria di stato di casa reale" dell'Archivio di Stato di Napoli (549. 1269/27), in cui si richiede a nome di Giovanna Campilli, "prima ballerina" del Teatro di San Carlo, una serata a suo beneficio; serata che otterrà poiché altre danzatrici successivamente ne fanno menzione. 
come quella coreografica dai soli programmi del ballo, che - come già accennato - ricalcavano nei soggetti, ma anche nella struttura narrativa, quelli precedenti a cui attingevano, mentre originale doveva essere di volta in volta la realizzazione scenica.

Certamente nei primi anni dell'Ottocento Giovanna Campilli come compositrice di balli rappresentò, qualunque fosse stato il suo stile coreografico, un'eccezione alla norma, che vedeva ancora per tutto il XIX secolo i soli uomini protagonisti della scrittura in movimento. E, nonostante un inizio entusiasmante con le coreografe Bronislava Nižinskaja, Agnes de Mille e Ninette de Valois, ancora nel Novecento le maggiori compagnie di balletto nazionali sono state caratterizzate da una predominante presenza maschile, sia per quanto concerne l'ambito creativo che quello della direzione artistica, diversamente da ciò che è avvenuto nell'ambito della danza moderna, dove si è verificato esattamente l'opposto, ovvero una prevalenza di coreografia al femminile. Ancora oggi l'argomento è attuale; non a caso nel 2012 la rivista "Dance Chronicle" ha lanciato un dibattitto sul tema "Where Are All the Women Choreographers in Ballet?" sull'onda di una nota affermazione del coreografo George Balanchine: «The principle of classical ballet is woman» (Meglin, Matluck Brooks, 2012, p. 1).

\section{BIBLIOGRAFIA}

Programmi dei balli:

(1780). La filosofia delle donne, balletto allegorico, per il Nobile Teatro Giustiniani di S. Moisè In Venezia, Inventato, e posto in su la Scena da Madama Anna Binetti il Carnovale dell'Anno 1780. Venezia: sine editione.

(1797). Oscar e Malvina, Ballo Eroico Pantomimo, In cinque Atti, composto e diretto da Mad. Maria De' Caro, Prima Ballerina, il Carnovale dell'Anno 1797, in Bianca De' Rossi, Dramma Nuovo per Musica da rappresentarsi Nel Nobilissimo Teatro Venier in San Benedetto [...]. Venezia: Modesto Fenzo.

(1810). Ippolita regina delle amazzoni, ballo eroico tragico-pantomimo, Inventato, e diretto da Giovanna Campilli, in I puntigli per equivoco 
dramma giocoso per musica da rappresentarsi nel Real Teatro Carolino [...]. Palermo: Felicella.

(1816). Ippolita regina delle amazzoni, ballo eroico Tragico-pantomimo, inventato, e diretto dalla signora Giovanna Campilli, Rappresentato la prima volta in Napoli nel real teatro del Fondo a' 17. Giugno 1816. Napoli: dalla tipografia Flautina.

(1827). Venere benefica, ballo favoloso pantomimo in quattro atti composto e diretto da Giovanna Campilli, Prima ballerina delle parti de' reali teatri. Rappresentato la prima volta nel real teatro del Fondo A' 19. Agosto 1827. Ricorrendo il fausto giorno natalizio di sua maestà Francesco I, re del Regno delle due Sicilie. Napoli: dalla tipografia Flautina.

\section{Testi:}

Bennahum, J. (2004). The Lure of Perfection: Fashion and Ballet, 1780-1830. London: Taylor \& Francis.

Casanova, G. (1965). Storia della mia vita (voll. 1-7), a cura di Piero Chiara. Milano: Arnoldo Mondadori Editore.

Christout, M.F. (1959). Le Picq, Charles. In Enciclopedia dello Spettacolo (vol. 6, coll. 1409-1410). Roma: Le Maschere.

Costa, M.R. (1987). De Caro. In Dizionario Biografico degli Italiani, vol. 33. Roma: Istituto della Enciclopedia italiana Treccani. Retrieved from http://www.treccani.it/enciclopedia/giulia-de-caro_(Dizionario-Biografico)/.

Croce, B. (1891). I teatri di Napoli. Napoli: Luigi Pierro.

Dorvane, J. (1998). Le Picq, Charles. In S.J. Cohen (Ed.), International Encyclopedia of Dance (vol. 4, pp. 149-150). New York: Oxford University Press.

Marquié, H., \& Nordera, M. (Eds.). (2015). Perspectives genres sur les femmes dans l'histoire de la danse. Recherches en Danse, 3. Retrieved from https://journals.openedition.org/danse/837.

Maresca, R. (1997). Le scuole di ballo del Teatro San Carlo dal 1812 al 1840: i documenti dell'Archivio Storico di Napoli. Chorégraphie, 10, 85-112.

Massaro, M.N. (1985). Il Ballo pantomimo al Teatro Nuovo di Padova (1751-1830). Acta Musicologica, LVII, 215-275.

Matluck Brooks, L. (Ed.). (2007). Women's Work. Making Dance in Europe Before 1800. Madison: The University of Wisconsin Press. 
McCleave, S. (2011). Danzatori italiani a Londra nel Settecento. In J. Sasportes (Ed.), La danza italiana in Europa nel Settecento (pp. 63-135). Roma: Bulzoni.

Meglin, J.A., \& Matluck Brooks, L. (2012). Where Are All the Women Choreographers in Ballet? Dance Chronicle, 35, 1-7.

Nordera, M. (2005). Generi in corso. Note per storie ancora da scrivere. In S. Franco \& M. Nordera (Eds.), I discorsi della danza. Parole chiave per una metodologia della ricerca (pp. 203-226). Torino: Utet.

Nordera, M. (2007). Come si costruisce una ballerina. Marie Madeleine Guimard tra vita e scena nelle fonti del XIX secolo. Teatro e Storia, 28, 381-393.

Noverre, J.-G. (2011). Lettere sulla danza, sui balletti e sulle arti (1803), a cura di F. Pappacena, trad. it. di A. Alberti. Lucca: LIM.

Onesti, S. (2016). Di passi, di storie e di passioni: teorie e pratiche del ballo teatrale nel secondo Settecento italiano. Torino: Accademia University Press.

Palumbo, R. (Ed.). (1877). Maria Carolina, regina delle due Sicilie, suo carteggio con Lady Emma Hamilton, Documenti inediti. Napoli: Nicola Jovene.

Pappacena, F. (2009). La danza classica. Le origini. Roma-Bari: Laterza.

Pappacena, F. (2015). Per una storia della danza. Danza francese e/o italiana? Ripensare il Settecento. Acting Archives Review, 9, 84-156.

Pappacena, F. (2016). Storia della danza in Occidente (vol. 2, Il Settecento e l'Ottocento). Roma: Gremese.

Pappacena, F. (2017). Il "libretto" come fonte di documentazione. In P. Veroli (Ed.), Il libretto di ballo. Riflessioni storiche e teoriche in omaggio ad Alberto Testa (pp. 3-16). Bologna: Massimiliano Piretti.

Piperno, F. (2017). Cesarotti all'opera: appunti sulla librettistica ossianica. In A. Rostagno \& S. Tatti (Eds.), Scrittori in Musica. I Classici Italiani nel Melodramma tra Seicento e Novecento (pp. 137-159). Roma: Bulzoni.

Ritorni, C. (1838). Commentarii della vita e delle opere coredrammatiche di Salvatore Viganò e della coregrafia e de' corepei. Milano: Guglielmini e Redaelli.

Ruffin, E., \& Trentin, G. (Eds.). (1994). Catalogo generale cronologico dei balli teatrali a Venezia. In J. Sasportes, E. Ruffin \& G. Trentin (Eds.) Balli Teatrali a Venezia (1746-1859). Partiture di sei balli pantomimici di 
Brighenti, Angiolini e O. Viganò (vol. 1, pp. XXXI-CCCXIX). Milano: Ricordi.

Ruffin, E. (1998). La prima traduzione italiana delle Lettres di Noverre: Venezia 1794. La danza italiana, 1, 35-58.

Sartori, C. (1990-1994). I libretti italiani a stampa dalle origini al 1800 (voll. 1-7). Cuneo: Bertola \& Locatelli Musica.

Scafidi, N. (1998). La danza alla Scala. In R. Albano, N. Scafidi \& R. Zambon, La danza in Italia. La Scala, La Fenice, il San Carlo dal XVIII secolo ai giorni nostri (pp. 9-87). Roma: Gremese.

Tiby, O. (1957). Il real teatro carolino e l'Ottocento musicale palermitano. Firenze: Leo S. Olschki.

Wiel, T. (1897). I teatri musicali veneziani del Settecento. Catalogo delle opere in musica rappresentate nel secolo XVIII in Venezia. Venezia: Fratelli Visentini.

Winter, M.H. (1974). The Pre-Romantic Ballet. London: Pitman.

Zambon, R. (1997). Il «grido della natura»: il teatro di danza alla fine del Settecento a Venezia. In A. Bescinelli (Ed.), Naturale e artificiale in scena nel secondo Settecento (pp. 253-264). Roma: Bulzoni.

\footnotetext{
Riassunto: Il saggio descrive alcuni aspetti della vita e dell'attività artistica di Anna Binetti, Maria De Caro e Giovanna Campilli, "prime ballerine" vissute fra la seconda metà del Settecento e l'inizio dell'Ottocento, con il fine di mettere in luce la loro pratica di coreografe in un'epoca che vedeva questo mestiere riservato per lo più agli uomini. Attraverso i programmi dei balli e altre fonti coeve, il saggio documenta un ambito poco esplorato dagli studi di danza - ovvero la presenza femminile come "soggetto artistico" nel teatro di danza italiano - contestualizzando tale fenomeno nel più ampio panorama culturale dell'epoca e focalizzando l'attenzione su tre casi di donne-coreografe che, se pur isolati e straordinari, possono trovare delle convergenze significative nei luoghi della rappresentazione, Venezia e Napoli in particolare, e in alcune figure, come Jean-Georges Noverre e Maria Carolina d'Asburgo, interessanti a evidenziare possibili reti di rapporti verso cui aprire nuove prospettive di ricerca.
}

Parole chiave: Anna Binetti, Maria De Caro, Giovanna Campilli, ballo pantomimo italiano del XVIII-XIX secolo, programmi/libretti di ballo 